Artículo

\title{
Código de SAS para analizar un dialelico completo y heterosis. Un ambiente
}

Delfina de Jesús Pérez López

Claudia Saavedra Guevara

Martín Rubí Arriaga

J. Ramón Pascual Franco Martínez

Francisco Gutiérrez Rodríguez

Andrés González Huerta ${ }^{\S}$

Centro de Investigación y Estudios Avanzados en Fitomeroramiento-Facultad de Ciencias AgrícolasUniversidad Autónoma del Estado de México-Campus Universitario 'El Cerrillo'. El Cerrillo Piedras Blancas, Toluca, Estado de México, México. AP. 435. Tel. 722 2965531, ext. 148. (djperezl@uaemex.mx; csg1003@yahoo.com; m_rubi65@yahoo.com.mx; jrfrancom@uaemex.mx; fgrfca@ hotmail.com.

${ }^{\S}$ Autor para correspondencia: agonzalezh@uaemex.mx.

\section{Resumen}

La elaboración de programas para sistema para análisis estadístico (SAS) y su validación con softwares disponibles gratuitamente es indispensable cuando no existen recursos económicos para adquirir la licencia de un paquete estadístico apropiado. En este estudio se presenta un código para SAS y se realiza su validación con el programa propuesto por Zhang y Kang (1997), modificado por Saavedra (2019). El código genera un análisis de varianza con partición de los efectos de tratamientos en progenitores $(\mathrm{P})$, cruzas directas $(\mathrm{CD})$, cruzas recíprocas $(\mathrm{CR}), \mathrm{P}$ vs cruzas, y $\mathrm{CD}$ vs CR. Además de generar la comparación de medias de tratamientos con la prueba de Tukey, se estiman los efectos genéticos para progenitores o para sus cruzas (Gi, Sij, Rij, Mi); así como, los de heterosis con la media de ambos padres o con el mejor de ellos. Debido a que ambos códigos sólo coinciden en el cálculo de los efectos genéticos previamente indicados, se sugiere su aplicación simultánea para realizar un análisis completo del método 1 de Griffing (1956a, b). El código que ha sido propuesto será de gran utilidad para fitomejoradores y genetistas y especialmente, para estudiantes en ciencias biológicas y agropecuarias de nivel licenciatura y de postgrado con poco entrenamiento en el lenguaje de programación en SAS.

Palabras clave: bloques completos al azar, método 1 de Griffing, modelo 1, prueba de Tukey.

Recibido: febrero de 2020

Aceptado: abril de 2020 


\section{Introducción}

Las cruzas dialélicas fueron diseñadas antes de los 50's, pero pronto se convirtieron en una poderosa herramienta para mejoradores de plantas y animales, quienes para reconocer el mérito de varios progenitores evaluaron sus progenies a través de los efectos y varianzas de aptitud combinatoria general (ACG) y específica (ACE) (Sprague y Tatum, 1942; Griffing, 1956a, b; González et al., 2007a, b). Con éstas se definen nuevos patrones heteróticos o una población segregante a partir de la cual es posible aislar nuevamente plantas sobresalientes, predecir la respuesta a la selección o el comportamiento de híbridos o sintéticos formados con nuevas líneas (Hallauer y Miranda, 1988; Christie y Shattuck, 1992; González et al., 2007a, b).

El análisis de un experimento de cruzas dialélicas completo sin una computadora personal (PC) es laborioso y para ahorrar tiempo, existen varios paquetes estadísticos como SAS (https://www.sas.com/store/index.ep), Excel (Microsoft Office), Indostat (https://www.indostat.org), AGD-R (https://data.cimmyt.org/dataset.xhtml?persistentld= hdl:11529/ 10202), Agrobase II, generación (http://www.agronomix.com), PB Tools (https://pbtools.software.informer.com/2.0/), TNAUSTAT (https:// sites. google.com/site/tnaustat) y GSCA (https://bioseqdata.com/gsca/gsca.htm), entro otros; de éstos sólo Agrobase II generación e Indostat deben adquirirse con licencia con un costo superior a \$1 000.0 USD, debido a que se requieren al menos tres módulos para operar adecuadamente ambos softwares. Aunque SAS es el mejor paquete estadístico, es común que los mejoradores y genetistas utilicen varios softwares para analizar los datos de experimentos diseñados en las ciencias agropecuarias y biológicas (Padilla $e t$ al., 2019a; Padilla et al., 2019b; Saavedra, 2019).

También, para muchos usuarios es difícil descargar software gratuito debido a que hay problema de incompatibilidad entre éste y sus PC's, existen problemas técnicos durante las descargas, no se obtiene el permiso necesario, los investigadores no responden las solicitudes o el programa no funciona en versiones antiguas o recientes de Windows. En este contexto, sería deseable elaborar y validar algunos códigos para SAS, para versiones 6.01 o superiores (SAS, 1989), que permitan complementar el análisis genético-estadístico para experimentos de cruzas dialélicas completas.

\section{Materiales y métodos}

\section{Dialélico completo}

En la metodología 1, descrita en Saavedra (2019), el análisis de varianza (ANOVA) para un solo ambiente contiene repeticiones (R), tratamientos (Trat) y error experimental, su modelo estadístico corresponde a un diseño de bloques completos al azar. En el ANOVA, los efectos de Trat se dividen en progenitores $(\mathrm{P})$, cruzas directas (CD), cruzas recíprocas (CR), $\mathrm{P}$ vs cruzas y $\mathrm{CD}$ vs $\mathrm{CR}$, como lo sugirió González et al. (2007b), ambos contrastes estiman heterosis promedio y efectos maternos y no maternos.

El programa calcula las diferencias entre Trat con la prueba de Tukey (SAS, 1989). Este código puede modificarse fácilmente si el usuario requiere de otras pruebas de comparación de medias, o de diversos análisis de regresión y correlación, estos análisis pueden extenderse a series de experimentos en tiempo y espacio (Saavedra, 2019). 
En la metodología 2, que corresponde al método 1 de Griffing (1956a, b), el ANOVA para un solo ensayo tiene repeticiones (R), aptitud combinatoria general (ACG), aptitud combinatoria específica (ACE), efectos maternos (EM) y efectos recíprocos (ER); en las series de experimentos podrían estimarse las interacciones de éstos con sólo dos ambientes.

También, en ambos casos, serían estimables los efectos de gi para cada progenitor o de sij para cada cruza, los efectos recíprocos y maternos (Zhang y Kang, 1997). Los componentes de varianza y heredabilidades, y la predicción de híbridos y sintéticos podrían estimarse con otros programas para SAS (Martínez, 1983; González et al., 2007a, b; Montesinos et al., 2007).

\section{Definición de variables en el código}

En la base de datos denominada 'dialelo' se define female, male, YH, YP, YM, X, Y, A, B, C, D y M, en las variables female y male se indican las combinaciones de cada hembra con cada macho, YH, YP y YM corresponden a las medias de cruza, hembra y macho, respectivamente. En X, Y se capturan los totales para cada pareja de CD y CR. Después de realizado la suma sobre repeticiones en A, B, C, D, cada línea de la CD o de CR aparece dos veces, como hembra y como macho (Yi. ó Y.i.; Yj.. o Y.j.)M es la gran media aritmética, GI, SIJ, RIJ y MI son los mismos efectos genéticos que se estiman con las fórmulas propuestas en el método 1 de Griffing (1956a, b).

\section{Valores usados en el código}

En este estudio fueron utilizados 96 datos, correspondientes a cuatro progenitores, sus seis cruzas directas y sus seis cruzas recíprocas, registrados en seis repeticiones (Saavedra, 2019).

\section{Resultados y discusión}

Desde su creación en 1972, los programas para SAS para el análisis de experimentos de cruzas dialélicas han sido implementados en computadoras personales PC's por varios investigadores. Los grandes logros que se han obtenido para PC's se atribuyen a Schaffer y Usanis (1989); Burow y Coors (1994); Magari y Kang (1994); Zhang y Kang (1997); Martínez (1983, 1991), entre otros. Más recientemente, Mastache y Martínez (1998a, 1998b, 1999a, 1999b), perfeccionaron sus algoritmos para obtener los mejores predictores lineales e insesgados (MPLI) empíricos de los efectos de los progenitores, para ayudar a los usuarios con poco entrenamiento en programación, cuando se utilizan diseños completamente al azar (DCA) y bloques completos al azar (BCA).

También, Mastache y Martínez (2003) obtuvieron un algoritmo integrado para su análisis simultáneo en experimentos balanceados para modelos de efectos fijos o aleatorios. Estos y otros programas podrían emplearse también para validar y para complementar las salidas que se obtuvieron con el código que se propone en el presente estudio (Zhang et al., 2005; Montesinos et al., 2007).

Zhang et al. (2005) modificaron los códigos de Zhang y Kang (1997); en Diallel-SAS05, ellos discutieron un programa más eficiente para el análisis genético-estadístico de los cuatro métodos de Griffing (1956a, b), incluyendo los correspondientes a los diseños II y III de Gardner y Eberhart (1966). Este programa es más amigable y es más fácil de modificar que Diallel-SAS, cuando los 
progenitores varían de 4 a 12, cuando no hay restricción en el número de ambientes, y cuando se estiman los efectos y varianzas de ACG y ACE para progenitores y cruzas, así como de sus interacciones con ambientes. Al igual que con otros paquetes estadísticos, hay problemas para implementarse en computadoras personales con versiones recientes de Windows (Padilla et al., 2019a, b).

Con el programa 1a se calcula el ANOVA y la comparación de medias (Tukey, $p=0.01$ ). Como Trat y sus componentes se consideran como efectos fijos, las pruebas de $\mathrm{F}$ se prueban con el cuadrado medio del error experimental o residual del modelo. En su código, se utiliza Data, SET, IF-THEN, ANOVA y GLM para definir subconjuntos de datos. El usuario será cuidadoso en respetar el orden correcto en la base de datos: P, CD y CR, los signos y los coeficientes de los contrastes, al igual que para otros paquetes estadísticos, deben capturarse dentro del programa. Si hay alguna duda para diseñar este tipo de contrastes se sugiere consultar a Padilla et al. (2019a).

En la salida del SAS, si $\mathrm{R}=6$ y Trat= 4, el ANOVA corresponde a progenitores; su prueba de hipótesis no es correcta, debido a que se construyó como un subconjunto y su cuadrado medio del residual es una fracción de los 96 datos. En este contexto deberá consultarse una tabla de F en esta etapa no hay restricciones con relación al número de variables por analizar. El código puede modificarse para incluir las pruebas de la diferencia mínima significativa (DMS o LSD), Dunnett, o contrastes mutuamente ortogonales, entre otras. Con dos o más variables, es posible modificar el programa para realizar regresión y correlación, estimar estadísticos simples y aplicar metodologías multivariadas, entre otros.

El código correspondiente al programa 1a se presenta a continuación:

Data maiz; Input rep trat PVG;Cards;

101758

102761

615768

616758 ;

DATA PADRES;SET MAIZ;IF TRAT>4 THEN DELETE;*'sólo progenitores;

DATA CD;SET MAIZ;IF TRAT<5 OR TRAT>10 THEN DELETE;*'sólo cruzas directas;

DATA CR; SET MAIZ; IF TRAT<11 THEN DELETE; *sólo cruzas recíprocas;

PROC ANOVA DATA=MAIZ; CLASS REP TRAT; MODEL PVG=REP TRAT; MEANS

TRAT/TUKEY LINES ALPHA=0.01;*Análisis con los 96 datos;

PROC GLM DATA=MAIZ; CLASS REP TRAT; MODEL PVG=REP TRAT;

CONTRAST "P VS CRUZAS"TRAT 121212 12 -4 -4 -4 -4 -4 -4 -4 -4 -4 -4 -4 -4;

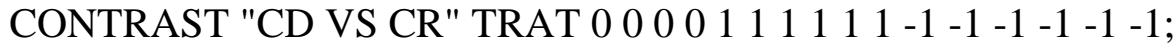

PROC ANOVA DATA=PADRES; CLASS REP TRAT; MODEL PVG=REP TRAT;*Análisis de varianza para progenitores;

PROC ANOVA DATA=CD; CLASS REP TRAT; MODEL PVG=REP TRAT;*Análisis de varianza para cruzas directas;

PROC ANOVA DATA=CR; CLASS REP TRAT; MODEL PVG=REP TRAT;*Análisis de varianza para cruzas recíprocas; RUN;

Con el programa $1 \mathrm{~b}$ se obtienen las estimaciones de los efectos genéticos (Gi, Sij, Rij, MI) y la heterosis (\%). La definición de variables antes de CARDS debe indicarse correctamente utilizando los datos de los Cuadros 1 y 2 (Saavedra, 2019), pero deben corregirse los valores del denominador 
de las fórmulas de GI, SIJ, RIJ y MI, si cambia R, P o ambos. En esta etapa es fundamental recurrir a los artificios que Martínez (1983) utilizó estableciendo una forma lógica de relacionar las fórmulas de Griffing (1956a, b) con el lenguaje de programación en SAS (SAS Institute, 1989).

Cuadro 1. Peso volumétrico del grano $\left(\mathrm{g} \mathrm{L}^{-1}\right)$ de 16 cruzas formadas con cuatro líneas.

\begin{tabular}{ccccccccc}
\hline Cruza & R1 & R2 & R3 & R4 & R5 & R6 & Total & Media \\
\hline 1) $1 \times 1$ & 758 & 734 & 750 & 790 & 758 & 765 & 4555 & 759.1 \\
2) $2 \times 2$ & 761 & 762 & 737 & 779 & 763 & 773 & 4575 & 762.5 \\
3) $3 \times 3$ & 802 & 812 & 802 & 838 & 793 & 782 & 4829 & 804.8 \\
4) $4 \times 4$ & 790 & 768 & 780 & 772 & 783 & 775 & 4668 & 778 \\
5) $1 \times 2$ & 814 & 792 & 770 & 781 & 775 & 755 & 4687 & 781.1 \\
6) $1 \times 3$ & 805 & 803 & 806 & 832 & 813 & 824 & 4883 & 813.8 \\
7) $1 \times 4$ & 791 & 775 & 777 & 791 & 795 & 780 & 4709 & 784.8 \\
8) $2 \times 3$ & 819 & 816 & 793 & 814 & 818 & 786 & 4846 & 807.6 \\
9) $2 \times 4$ & 779 & 778 & 758 & 798 & 783 & 755 & 4651 & 775.1 \\
10) $3 \times 4$ & 830 & 830 & 850 & 853 & 828 & 806 & 4997 & 832.8 \\
11) $2 \times 1$ & 774 & 772 & 786 & 750 & 794 & 769 & 4645 & 774.16 \\
12) $3 \times 1$ & 789 & 808 & 816 & 808 & 824 & 806 & 4851 & 808.5 \\
13) $4 \times 1$ & 787 & 815 & 815 & 825 & 802 & 796 & 4840 & 806.6 \\
14) $3 \times 2$ & 817 & 832 & 808 & 775 & 790 & 797 & 4819 & 803.1 \\
15) $4 \times 2$ & 756 & 768 & 756 & 754 & 753 & 768 & 4555 & 759.1 \\
16) $4 \times 3$ & 850 & 820 & 840 & 850 & 805 & 758 & 4923 & 820.5 \\
Total & 12722 & 12865 & 12644 & 12810 & 12677 & 12495 & 76033 & 792.01 \\
\hline
\end{tabular}

Cuadro 2. Valores usados para estimar efectos genéticos y heterosis.

\begin{tabular}{cccccc}
\hline & 1 & 2 & 3 & 4 & Total \\
\hline 1 & 4555 & 4687 & 4883 & 4709 & 18834 \\
2 & 4645 & 4575 & 4846 & 4651 & 18717 \\
3 & 4851 & 4819 & 4829 & 4997 & 19496 \\
4 & 4840 & 4555 & 4923 & 4668 & 18986 \\
Total & 18891 & 18636 & 19481 & 19025 & 76033 \\
\hline
\end{tabular}

Nota: se sumó sobre repeticiones y los totales de hilera o de columna son la contribución de cada línea hembra o macho, respectivamente.

En algunas columnas como la gran media aritmética (M), que es una constante para los 12 apareamientos, hay valores duplicados, pero es fácil establecer a que progenitor o cruza corresponden porque los datos se muestran descendentes (González et al., 2007a, b; Saavedra, 2019).

El código correspondiente al programa $1 b$ se presenta a continuación:

DATA HETERO; INPUT FEMALE MALE YH YP YM X Y A B C D M;

$\mathrm{MP}=\mathrm{YP}+\mathrm{YM}) / 2 ; *$ para calcular la media de los padres $(\mathrm{MP})$;

$\mathrm{BP}=\mathrm{MAX}(\mathrm{YP}, \mathrm{YM}) ; *$ para elegir al mejor progenitor $(\mathrm{BP})$;

$\mathrm{DMP}=\mathrm{YH}-\mathrm{MP}$; ${ }^{*}$ para estimar el numerador de la fórmula de heterosis con MP;

$\mathrm{HMP}=(\mathrm{DMP} / \mathrm{MP}) * 100 ;{ }^{*}$ para estimar heterosis con la media de los padres, en \%; 
$\mathrm{DBP}=\mathrm{YH}-\mathrm{BP} ;{ }^{*}$ calcula el numerador de la fórmula de heterosis con BP;

$\mathrm{HBP}=(\mathrm{DBP} / \mathrm{BP}) * 100$; ${ }^{*}$ calcula heterosis con el mejor padre, en \%;

$\mathrm{GI}=(\mathrm{A}+\mathrm{B}) / 48-\mathrm{M}$; *estima los efectos de gi;

$\mathrm{SIJ}=(\mathrm{X}+\mathrm{Y}) / 12-(\mathrm{A}+\mathrm{B}+\mathrm{C}+\mathrm{D}) / 48+\mathrm{M} ;{ }^{*}$ calcula los efectos de $\mathrm{Sij}$;

$\mathrm{RIJ}=(\mathrm{X}-\mathrm{Y}) / 12 ; *$ determina los efectos de rij;

$\mathrm{MI}=(\mathrm{A}-\mathrm{B}) / 48 ;{ }^{*}$ calcula los efectos mi;

CARDS;

12781.1759 .1762 .54687464518834188911871718636792 .01

13813.8759 .1804 .84883485118834188911949619481792 .01

14784.8759 .1778 .04709484018834188911898619025792 .01

23807.6762 .5804 .84846481918717186361949619481792 .01

24775.1762 .5778 .04651455518717186361898619025792 .01

34832.8804 .8778 .04997492319496194811898619025792 .01

21774.1762 .5759 .14645468718636187171889118834792 .01

31808.5804 .8759 .14851488319481194961889118834792 .01

41806.6778 .0759 .14840470919025189861889118834792 .01

32803.1804 .8762 .54819484619481194961863618717792 .01

42759.1778 .0762 .54555465119025189861863618717792 .01

43820.5778 .0804 .84923499719025189861948119496792 .01

TITLE 'Efectos de gi, sij, rij, mi y heterosis para el dialélico general';

DATA DOS; SET HETERO; PROC PRINT; RUN;

Procedimiento ANOVA

Variable dependiente: PVG

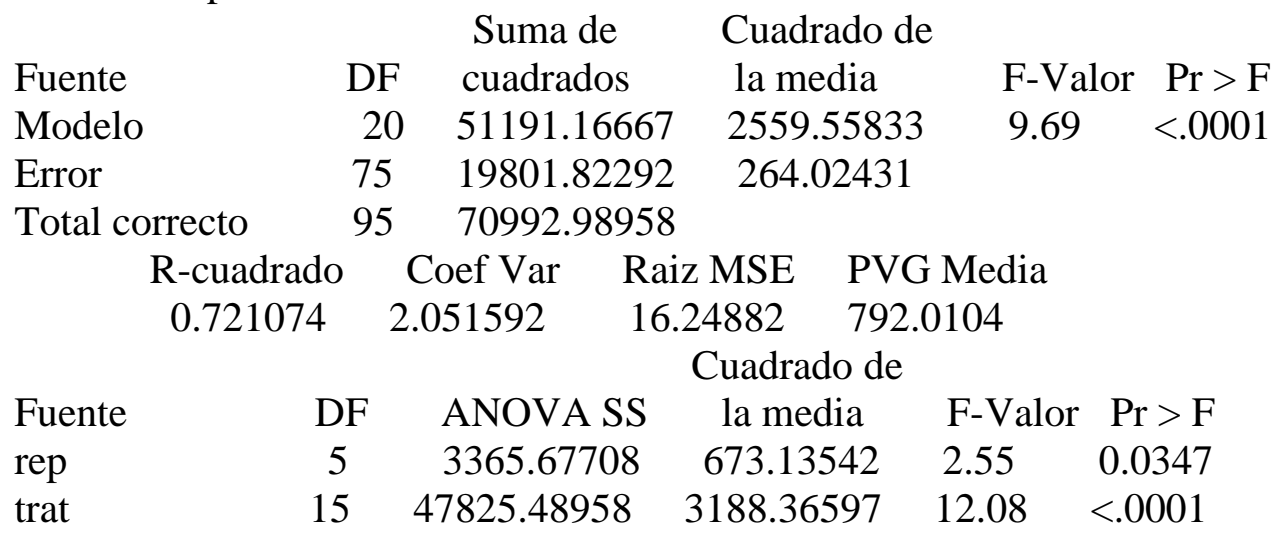

Prueba del rango estudentizado de Tukey (HSD) para PVG

NOTA: este test controla el índice de error experimentwise de tipo I, pero normalmente tiene un índice de error de tipo II más elevado que REGWQ.

$\begin{array}{ll}\text { Alfa } & 0.01 \\ \text { Error de grados de libertad } & 75 \\ \text { Error de cuadrado medio } & 264.0243 \\ \text { Valor crítico del rango estudentizado } & 5.76634 \\ \text { Diferencia significativa mínima } & 38.251\end{array}$

Medias con la misma letra no son significativamente diferentes. 


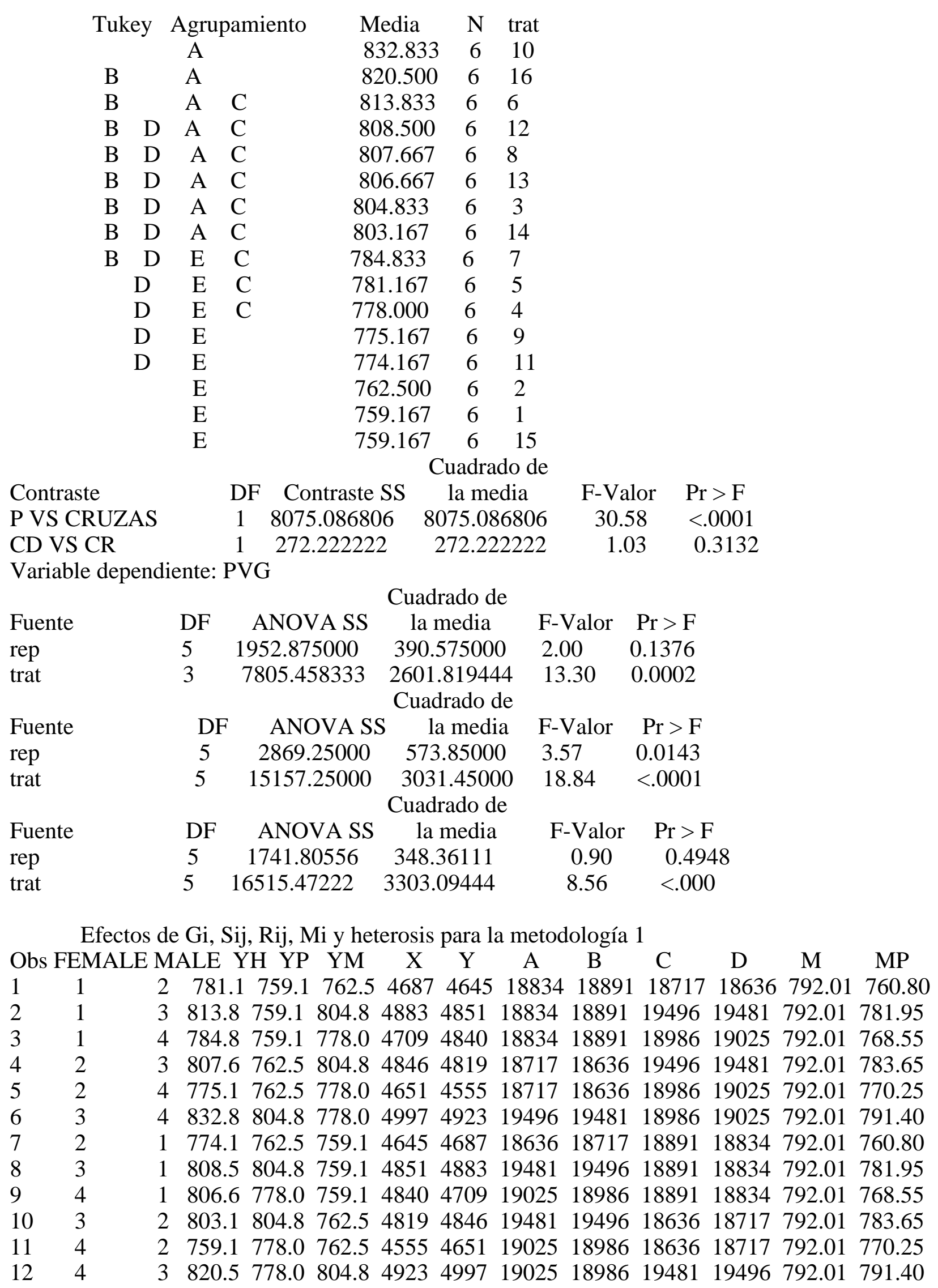




$\begin{array}{cccccccccc}\text { Obs } & \text { BP } & \text { DMP } & \text { HMP } & \text { DBP } & \text { HBP } & \text { GI } & \text { SIJ } & \text { RIJ } & \text { MI } \\ 1 & 762.5 & 20.30 & 2.66824 & 18.6 & 2.43934 & -6.0725 & 5.5517 & 3.5000 & -1.1875 \\ 2 & 804.8 & 31.85 & 4.07315 & 9.0 & 1.11829 & -6.0725 & 5.2183 & 2.6667 & -1.1875 \\ 3 & 778.0 & 16.25 & 2.11437 & 6.8 & 0.87404 & -6.0725 & 9.9267 & -10.9167 & -1.1875 \\ 4 & 804.8 & 23.95 & 3.05621 & 2.8 & 0.34791 & -13.8225 & 7.2183 & 2.2500 & 1.6875 \\ 5 & 778.0 & 4.85 & 0.62967 & -2.9 & -0.37275 & -13.8225 & -10.9067 & 8.0000 & 1.6875 \\ 6 & 804.8 & 41.40 & 5.23124 & 28.0 & 3.47913 & 20.0108 & 14.7600 & 6.1667 & 0.3125 \\ 7 & 762.5 & 13.30 & 1.74816 & 11.6 & 1.52131 & -13.8225 & 5.5517 & -3.5000 & 1.6875 \\ 8 & 804.8 & 26.55 & 3.39536 & 3.7 & 0.45974 & 20.0108 & 5.2183 & -2.6667 & 0.3125 \\ 9 & 778.0 & 38.05 & 4.95088 & 28.6 & 3.67609 & -0.1142 & 9.9267 & 10.9167 & -0.8125 \\ 10 & 804.8 & 19.45 & 2.48198 & -1.7 & -0.21123 & 20.0108 & 7.2183 & -2.2500 & 0.3125 \\ 11 & 778.0 & -11.15 & -1.44758 & -18.9 & -2.42931 & -0.1142 & -10.9067 & -8.0000 & -0.8125 \\ 12 & 804.8 & 29.10 & 3.67703 & 15.7 & 1.95080 & -0.1142 & 14.7600 & -6.1667 & -0.8125\end{array}$

Los resultados anteriores fueron validados con el programa elaborado por Zhang y Kang (1997). En el ANOVA el código permite la partición de los efectos de cruzas posibles en ACG, ACE, ER y EM, cuando se implementaron SORT, BY, GLM, IF-THEN, DROP, ARRAY, ELSE, GLM, CONTRAST, ESTIMATE y algunas MACROS. En Martínez (1991) se presentan éstas y otras componentes para elaborar el código de referencia.

El programa de Zhang y Kang (1997) aplica a los cuatro métodos de Griffing (1956a, b), para el método 1 se analizan $\mathrm{m}$ variables en dos ambientes. En el presente estudio, éste se amoldó a un solo ambiente implementando la restricción IF ENV> 1 THEN DELETE o IF ENV $<2$ THEN DELETE, capturado antes de DROP y después del INPUT. Su modificación es más laboriosa para los usuarios con poco entrenamiento en programación y, especialmente cuando el análisis se extiende a series de experimentos (Singh, 1973; Mastache y Martínez, 2003; Zhang et al., 2005).

TNAUSTAT software, además de calcular los efectos genéticos relacionados con progenitores y con sus cruzas en el método 1 de Griffing (1956a, b) también, simultáneamente, permite el cálculo del vigor híbrido con la media de ambos padres, con el mejor de ellos y adicionalmente, con base a heterosis comercial. Éste tiene la ventaja adicional de estimar los parámetros genéticos correspondientes al diseño de apareamiento I, propuesto por Hayman (1954). Sin embargo, este software fue diseñado para funcionar adecuadamente en una plataforma con MS Dos, por lo que anticipadamente debe descargarse del internet DOSBox software.

El programa de Zhang y Kang (1997), modificado por Saavedra (2019), se presenta a continuación: OPTIONS PS=56 LS=78; TITLE 'METHOD 1'; DATA METHOD1;

INPUT I J REP HYBRID YIELD ENV; IF ENV>1 THEN DELETE;DROP N NI NJ P; $\mathrm{P}=4$;*NUMBER OF PARENTAL LINES? ; ARRAY GCA (N) G1 G2 G3;DO N=1 TO (P-1); $\mathrm{GCA}=((\mathrm{I}=\mathrm{N})-(\mathrm{I}=\mathrm{P}))+((\mathrm{J}=\mathrm{N})-(\mathrm{J}=\mathrm{P}))$; END;ARRAY SCA(N) S11 S12 S13 S22 S23 S33; $\mathrm{N}=0$; DO NI=1 TO (P-1); DO NJ=NI TO (P-1); N+1; IF NI=NJ THEN DO; $\mathrm{SCA}=(\mathrm{I}=\mathrm{NI}) *((\mathrm{~J}=\mathrm{NJ})-(\mathrm{J}=\mathrm{P}))+(\mathrm{I}=\mathrm{P}) *((\mathrm{~J}=\mathrm{P})-(\mathrm{J}=\mathrm{NI})) ; \mathrm{END}$;ELSE DO; $\mathrm{SCA}=(\mathrm{I}=\mathrm{NI}) *(\mathrm{~J}=\mathrm{NJ})-(\mathrm{J}=\mathrm{P}) *((\mathrm{I}=\mathrm{NI})+(\mathrm{I}=\mathrm{NJ})-(\mathrm{I}=\mathrm{P}) * 2)+(\mathrm{I}=\mathrm{NJ}) *(\mathrm{~J}=\mathrm{NI})$ $-(\mathrm{I}=\mathrm{P}) *((\mathrm{~J}=\mathrm{NI})+(\mathrm{J}=\mathrm{NJ}))$; END;END;END; ARRAY REC (N) R12 R13 R14 R23 R24 R34; N=0; DO NI=1 TO (P-1); DO NJ= $(\mathrm{NI}+1)$ TO P; $\mathrm{N}+1 ; \mathrm{REC}=(\mathrm{I}=\mathrm{NI}) *(\mathrm{~J}=\mathrm{NJ})-(\mathrm{j}=\mathrm{NI}) *(\mathrm{I}=\mathrm{NJ})$; END;END; ARRAY MAT (N) M1 M2 M3; DO N=1 TO (P-1); MAT= (I=N) + (J=P)-(J=N)-(I=P); 
END;ARRAY NONM (N) N12 N13 N23;N=0;DO NI=1 TO (P-2);DO NJ=(NI+1) TO (P$1) ; \mathrm{N}+1 ; \mathrm{NONM}=((\mathrm{I}=\mathrm{NI}) *(\mathrm{~J}=\mathrm{NJ}))-(\mathrm{I}=\mathrm{NJ}) *(\mathrm{~J}=\mathrm{NI})-((\mathrm{I}=\mathrm{NI}) *(\mathrm{~J}=\mathrm{P}))+(\mathrm{I}=\mathrm{NJ}) *(\mathrm{~J}=\mathrm{P})$ $+\left((\mathrm{I}=\mathrm{P})^{*}((\mathrm{~J}=\mathrm{NI})-(\mathrm{J}=\mathrm{NJ}))\right)$; END;END;CARDS;

111017581

121028141

131038051

436157581

446167751 ;

PROC SORT; BY REP ENV I J; PROC GLM; CLASS REP ENV HYBRID; MODEL YIELD=ENV REP(ENV) HYBRID HYBRID*ENV; TEST H=HYBRID $\mathrm{E}=\mathrm{HYBRID} * \mathrm{ENV}$;LSMEANS HYBRID;

RUN; TITLE 'DIALLEL-SAS 1'; PROC GLM; CLASS REP ENV HYBRID;

MODEL YIELD= ENV REP (ENV) G1 G2 G3 S11 S12 S13 S22 S23 S33 R12 R13 R14 R23 R24 R34 G1*ENV G2*ENV G3*ENV S11*ENV S12*ENV S13*ENV S22*ENV S23*ENV S33*ENV R12*ENV R13*ENV R14*ENV R23*ENV R24*ENV R34*ENV;

\%MACRO GCASCA; CONTRAST 'GCA' G1 1, G2 1, G3 1;

CONTRAST 'SCA' S11 1, S12 1, S13 1, S22 1, S23 1, S33 1;

ESTIMATE 'G1' G1 1; ESTIMATE 'G2' G2 1; ESTIMATE 'G3' G3 1;

Estimate 'G4' G1 -1 G2 -1 G3 -1;

ESTIMATE 'S11' S11 1; ESTIMATE 'S12' S12 1; ESTIMATE 'S13' S13 1;

ESTIMATE 'S22' S22 1; ESTIMATE 'S23' S23 1; ESTIMATE 'S33' S33 1;

Estimate 'S14' S11 -1 S12 -1 S13 -1;

Estimate 'S24' S12 -1 S22 -1 S23 -1;

Estimate 'S34' S13 -1 S23 -1 S33 -1;

Estimate 'S44' S11 1 S12 2 S13 2 S22 1 S23 2 S33 1;

$\%$ MEND GCASCA; \%GCASCA \%MACRO INTERACT;

CONTRAST 'GCA*ENV' G1*ENV 1 -1, G2*ENV 1 -1, G3*ENV 1 -1;

CONTRAST 'SCA*ENV' S11*ENV 1 -1, S12*ENV 1 -1, S13*ENV 1 -1, S22*ENV 1 -1,

S23*ENV 1 -1, S33*ENV 1 -1; \%MEND INTERACT; \%INTERACT

CONTRAST 'REC' R12 1, R13 1, R14 1, R23 1, R24 1, R34 1;

ESTIMATE 'R12' R12 1; ESTIMATE 'R13' R13 1; ESTIMATE 'R14' R14 1;

ESTIMATE 'R23' R23 1; Estimate 'R24' R24 1; ESTIMATE 'R34' R34 1;

CONTRAST 'REC*ENV' R12*ENV 1 -1,R13*ENV 1 -1,R14*ENV 1 -1,R23*ENV 1 -

1,R24*ENV $1-1, \mathrm{R} 34 * \mathrm{ENV} 1-1$;

CONTRAST 'MAT SS' R12 1 R13 1 R14 1, R12 -1 R23 1 R24 1, R13 -1 R23 -1 R34 1, R14 -1

R24 -1 R34 -1; ESTIMATE 'MAT1' R12 1 R13 1 R14 1/DIVISOR=3;

ESTIMATE 'MAT2' R12 -1 R23 1 R24 1/DIVISOR=3;

ESTIMATE 'MAT3' R13 -1 R23 -1 R34 1/DIVISOR=3;

ESTIMATE 'MAT4' R14 -1 R24 -1 R34 -1/DIVISOR=3; RUN;

TITLE 'DIALLEL-SAS 2'; PROC GLM; CLASS REP ENV HYBRID;

MODEL YIELD= ENV REP (ENV) G1 G2 G3 S11 S12 S13 S22 S23 S33

M1 M2 M3 N12 N13 N23 G1*ENV G2*ENV G3*ENV

S11*ENV S12*ENV S13*ENV S22*ENV S23*ENV S33*ENV

M1*ENV M2*ENV M3*ENV N12*ENV N13*ENV N23*ENV;

$\%$ GCASCA \%INTERACT

CONTRAST 'MAT SS' M1 1, M2 1, M3 1;

CONTRAST 'NONM SS' N12 1, N13 1, N23 1;

CONTRAST 'MAT*ENV' M1*ENV 1 -1, M2*ENV 1 -1, M3*ENV 1 -1; 
CONTRAST 'NONM*ENV' N12*ENV 1 -1, N13*ENV 1 -1, N23*ENV 1- 1;

ESTIMATE 'M1' M1 1; ESTIMATE 'M2' M2 1; ESTIMATE 'M3' M3 1;

Estimate 'M4' M1 -1 M2 -1 M3 -1;

ESTIMATE 'N12' N12 1; ESTIMATE 'N13' N13 1; ESTIMATE 'N23' N23 1;

Estimate 'N14' N12 -1 N13 -1;

Estimate 'N24' N12 1 N23 -1;

Estimate 'N34' N13 1 N23 1; RUN;

Para validar el código que se presenta en el programa 1a, se muestran algunos resultados que genera el programa de Zhang y Kang (1997).

Variable dependiente: YIELD

\section{Procedimiento GLM}

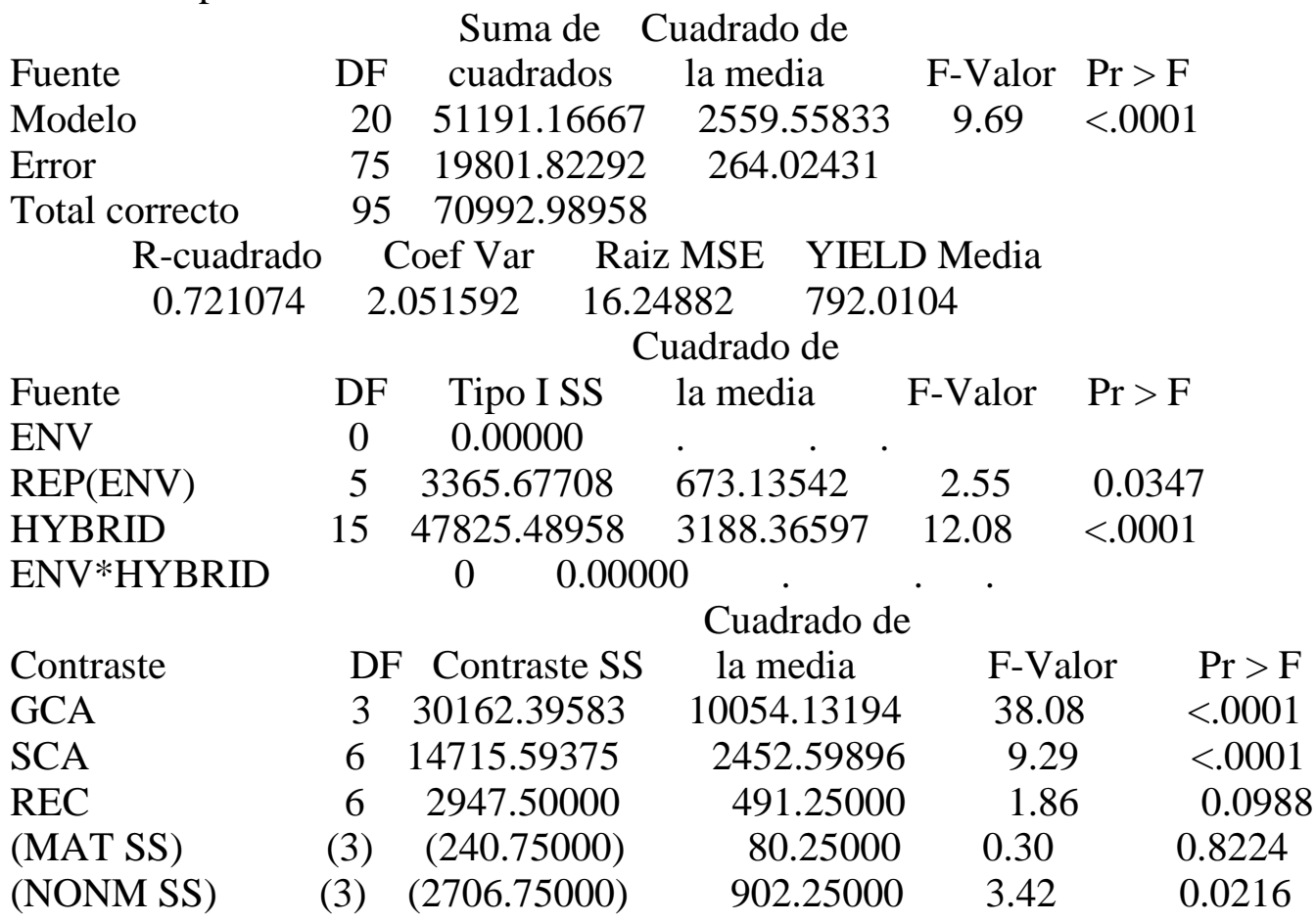

\begin{tabular}{lcccc} 
Parámetro & \multicolumn{4}{c}{$\begin{array}{c}\text { Estimación } \\
\text { estándar }\end{array}$} \\
G1 & -6.0729167 & 2.03110309 & -2.99 & 0.0038 \\
G2 & -13.8229167 & 2.03110309 & -6.81 & $<.0001$ \\
G3 & 20.0104167 & 2.03110309 & 9.85 & $<.0001$ \\
G4 & -0.1145833 & 2.03110309 & -0.06 & 0.9552 \\
S12 & 5.5520833 & 3.70826994 & 1.50 & 0.1385 \\
S13 & 5.2187500 & 3.70826994 & 1.41 & 0.1635 \\
S23 & 7.2187500 & 3.70826994 & 1.95 & 0.0553 \\
S14 & 9.9270833 & 3.70826994 & 2.68 & 0.0091 \\
S24 & -10.9062500 & 3.70826994 & -2.94 & 0.0043 \\
S34 & 14.7604167 & 3.70826994 & 3.98 & 0.0002 \\
M1 & -1.1875000 & 2.03110309 & -0.58 & 0.5605 \\
M2 & 1.6875000 & 2.03110309 & 0.83 & 0.4087 \\
M3 & 0.3125000 & 2.03110309 & 0.15 & 0.8781
\end{tabular}




$\begin{array}{lrccc}\text { M4 } & -0.8125000 & 2.03110309 & -0.40 & 0.6903 \\ \text { R12 } & 3.5000000 & 4.69063167 & 0.75 & 0.4579 \\ \text { R13 } & 2.6666667 & 4.69063167 & 0.57 & 0.5714 \\ \text { R14 } & -10.9166667 & 4.69063167 & -2.33 & 0.0226 \\ \text { R23 } & 2.2500000 & 4.69063167 & 0.48 & 0.6329 \\ \text { R24 } & 8.0000000 & 4.69063167 & 1.71 & 0.0922 \\ \text { R34 } & 6.1666667 & 4.69063167 & 1.31 & 0.1926\end{array}$

\section{Conclusiones}

Los programas ' $1 \mathrm{a}$ ' y ' $1 \mathrm{~b}$ ' son fáciles de utilizar y modificar para realizar un análisis de varianza en un solo ambiente, con la subdivisión de los efectos de los tratamientos en progenitores (P), cruzas directas $(\mathrm{CD})$, cruzas recíprocas $(\mathrm{CR}), \mathrm{P}$ versus cruzas y $\mathrm{CD}$ versus $\mathrm{CR}$. También es útil para realizar la comparación de medias de tratamientos (Tukey, $p=0.01$ ) y para estimar heterosis con la media de los padres y con el mejor de éstos cuando se analiza una variable.

El programa de Zhang y Kang (1997) fue diseñado para analizar ' $m$ ' variables, pero es más difícil de manipular cuando progenitores y ambientes es diferente de 5 y 2 , respectivamente. Por esta restricción, fue necesario modificar el código con $\mathrm{P}=4$. El código propuesto por Zhang y Kang estima aptitud combinatoria general y específica, efectos recíprocos y maternos, pero no incluye la prueba de Tukey ni la estimación de heterosis.

Los tres códigos para SAS corren en versiones de más de 10 años de su liberación comercial y en las académicas de prueba más recientes. El programa de Zhang y Kang (1997) permitió la validación confiable del código propuesto en el presente estudio cuando se estimaron los efectos genéticos, pero los tres códigos deben utilizarse para realizar un análisis dialélico más completo.

\section{Literatura citada}

Burow, M. D. and Coors, J. G. 1994. DIALLEL: a microcomputer program for the simulation and analysis of diallel crosses. Agron. J. 86(1):154-158.

Christie, B. R. and Shattuck, V. I. 1992. The diallel cross: design, analysis and use for plant breeders. Plant Breeding Reviews. 9(1):9-36.

Gardner, C. O. and Eberhart, S. A. 1966. Analysis and interpretation of the variety cross diallel and related populations. Biometrics. 22(3): 439-452.

González, H. A.; Sahagún, C. J. y Pérez, L. D. J. 2007a. Estudio de ocho líneas de maíz en un experimento dialélico incompleto. Ciencias Agrícolas Informa. 16(1):3-9.

González, H. A.; Pérez, L. D.; Sahagún, C. J.; Norman, M. T. H.; Balbuena, M. A. and Gutiérrez, R. F. 2007b. Análisis de una cruza dialélica completa de líneas endogámicas de maíz. Ciencias Agrícolas Informa. 16(1):10-17.

Griffing, B. 1956a. A generalized treatment of the use of diallel crosses in quantitative inheritance. Heredity. 10(1):31-50.

Griffing, B. 1956b. Concept of general and specific combining ability in relation to diallel crossing systems. Austr. J. Biol. Sci. 9(4):463-491.

Hallauer, A. R.; Miranda, F. O. J. B.1988. Quantitative Genetics in Maize Breeding. Iowa State University Press, Ames. Second Edition. USA. 468 p.

Hayman, B. I. 1954. The theory and analysis of the diallel crosses. Genetics. 39(1):798-809. 
Magari, R. and Kang, M. S. 1994. Interactive BASIC program for Griffing's diallel analysis. Journal of Heredity. 85(4):336.

Martínez, G. A. 1983. Diseño y análisis de los experimentos de cruzas dialélicas. Centro de Estadística y Cálculo. Colegio de Postgraduados. Chapingo, Estado de México. 252 p.

Martínez, G. A. 1991. Análisis de los experimentos dialélicos a través del procedimiento IML del SAS. Comunicaciones en Estadística y Cómputo. 10(2):1-36.

Mastache, L. A. A.; Martínez, G. A.; Castillo, M. A. y González, C. F. V. 1998a. Los mejores predictores lineales e insesgados (MPLI) en experimentos dialélicos parciales sin efectos maternos. Revista Fitotecnia Mexicana. 21(1):49-60.

Mastache, L. A. A.; Martínez, G. A. y Castillo, M. A. 1998b. Los mejores predictores lineales e insesgados (MPLI) en experimentos dialélicos parciales con efectos maternos. Revista Fitotecnia Mexicana. 21(2):171-184.

Mastache, L. A. A.; Martínez, G. A. y Castillo, M. A. 1999a. Los mejores predictores lineales e insesgados (MPLI) en los diseños dos y cuatro de Griffing. Agrociencia. 33(1):81-90.

Mastache, L. A. A.; Martínez, G. A. y Castillo, M. A. 1999b. Los mejores predictores lineales e insesgados (MPLI) en los diseños uno y tres de Griffing. Agrociencia. 33(3):349-359.

Mastache, L. A. A. y Martínez, G. A. 2003. Un algoritmo para el análisis, estimación y predicción en experimentos dialélicos balanceados. Revista Fitotecnia Mexicana. 26(3):191-200.

Montesinos, L. O. A.; Mastache, L. A. A.; Luna, E. I. y Hidalgo, C. J. V. 2007. Mejor predictor lineal e insesgado combinado para aptitud combinatoria general y análisis combinado de los diseños uno y tres de Griffing. Técnica Pecuaria en México. 45(2):131-146.

Padilla, L. A.; González, H. A.; Pérez, L. D. J.; Rubí, A. M.; Gutiérrez, R. F. y Franco, M. J. R. P. 2019a. InfoStat, InfoGen y SAS para contrastes mutuamente ortogonales en experimentos en bloques completos al azar en parcelas subdivididas. Rev. Mex. Cienc. Agríc. 10(6):1417-1431.

Padilla, L. A.; González, H. A.; Pérez, L. D. J.; Rubí, A. M.; Gutiérrez, R. F.; Ramírez, D. J. F.; Franco, M. J. R. P. y Serrato, C. R. 2019b. Programas para SAS e InfoStat para analizar una serie de experimentos en parcelas subdivididas. En: temas selectos en la innovación de las ciencias agropecuarias. Alfaomega Grupo Editor SA. de CV. Primera edición (Salgado y otros, eds.). México, DF. 724 p. ISBN: 9786075384115.

SAS Institute, Inc. 1989. SAS/IML software: Usage and reference. Version 6. First edition. Cary, N. C.

Saavedra, G. C. 2019. Estimación de parámetros genéticos en maíz con dos metodologías usando datos de una cruza dialélica completa. I. Un ambiente. Tesis de Maestro en Fitomejoramiento. Facultad de Ciencias Agrícolas, Universidad Autónoma del Estado de México. Toluca, Estado de México. 96 p.

Singh, D. 1973. Diallel analysis for combining ability over several environments-II. Indian Journal of Genetics and Plant Breeding. 33(1):469-481.

Schaffer, H. E. y Usanis, R. A. 1989. General least squares analysis of diallel experiments: A computer program. Genetics Dep. Res. Rep. 1. North Carolina State University, Raleigh. $61 \mathrm{p}$.

Sprague, G. F. and Tatum, L. A. 1942. General vs specific combining ability in single crosses of corn. J. Amer. Soc. Agron. 34(1):923-932.

Zhang, Y. and Kang, M. S. 1997. DIALLEL-SAS: A SAS program for Griffing's Diallel Analyses. Agronomy Journal. 89(2):176-182.

Zhang, Y.; Kang, M. S. and Lamkey, K. R. 2005. DIALLEL-SAS05: A comprehensive program for Griffing's and Gardner-Eberhart analysis. Agron. J. 97(4):1097-1106. 